\title{
Variations de l'hydraulicité en France
}

\section{River flow variations in France}

\author{
par M. COUTAGNE
}

INGENIEUR-CONSEIL, ANCIEN ĹLÈVE DE L'ĹCOLE POLYTEGHNJQUE

\begin{abstract}
La période trentenaire 1927-1956 se caractérise par un débit moyen normal. La Seine à Parts accuse les mêmes valeurs an cours de cetle série et de celle de 1851-1950. Ce fait est établi à partir des précipitations relevées à Auxerre et des bonnes corrélations pluviofluviales qui existent entre les résultats de ces deux stations. On a unalysé les deux époques humide et sèche des trenle années récentes dans le bassin de la Seine, puis dans les autres bassins français.
\end{abstract}

\begin{abstract}
The thirty yetr period from 1927 to 1956 is charricterized by normal average flow. Average flows for the Seine at Paris during this period are the same as for 1851 to 1950. This fact was determined from rainfall measurements made at Auxerre and correlations between these two stations for rainfall and river flow are good. The two wet and dry periods of the last thirty years are analysed for the Seine basin and then for other French river basins.
\end{abstract}

Il est évidemment intéressant de connaître la valeur moyenne et la variabilité des débits d'un bassin pendant une très longue période, mais c'est le plus souvent difficile.

Or, la série trentenaire 1927-1956 des débits de la Seine à Paris présente les mêmes chif- fres moyens annuels que la série séculaire 18511950 : c'est ce que montre la première partie.

Et cette règle, valable pour la Seine, est génćralisable aux autres grands bassins français ainsi qu'on le verra dans la deuxième partie.

\section{I. - DEBITS DE LA SEINE A PARIS DE 1851 à 1950}

\section{$1^{\circ}$ VARIATIONS DES PRÉcipitations QUINQUENNA- LEs a AuXerre.}

L'équivalence des deux séries de 30 et 100 ans est fondée sur les précipitations. A défaut d'une statisticque qui reste à établir, on considère une seule station témoin : Auxerre (Yonne).

Auxerre 1927-56=Auxerre $1851-1950=653 \mathrm{~mm}$

Pour les 50 ans de la période 1891-1940, les

(*) Cette étude est un résumé par J. de Braunegard, ingénieur à Electricité de France, de quatre notes de M. Aimé Coutagne. précipitations moyennes $d u$ bassin atteignent $728 \mathrm{~mm}$ (d'après les moyennes départementales établies par M. Grisollet) et celles d'Auxerre $674 \mathrm{~mm}$. On admet donc:

précipitations bassin $=$

précipitations Auxerre $\times 1,08(728 / 674)$

période centenaire 1851-1950 :

Auxerre . . . . . . . . . . $653 \mathrm{~mm}$ bassin $653 \times 1,08=\ldots \ldots \ldots \ldots 705 \mathrm{~mm}$

La figure 1 porte en ahscisse le temps, en ordonnée la pluviosité en $\%$ de la période cente- 


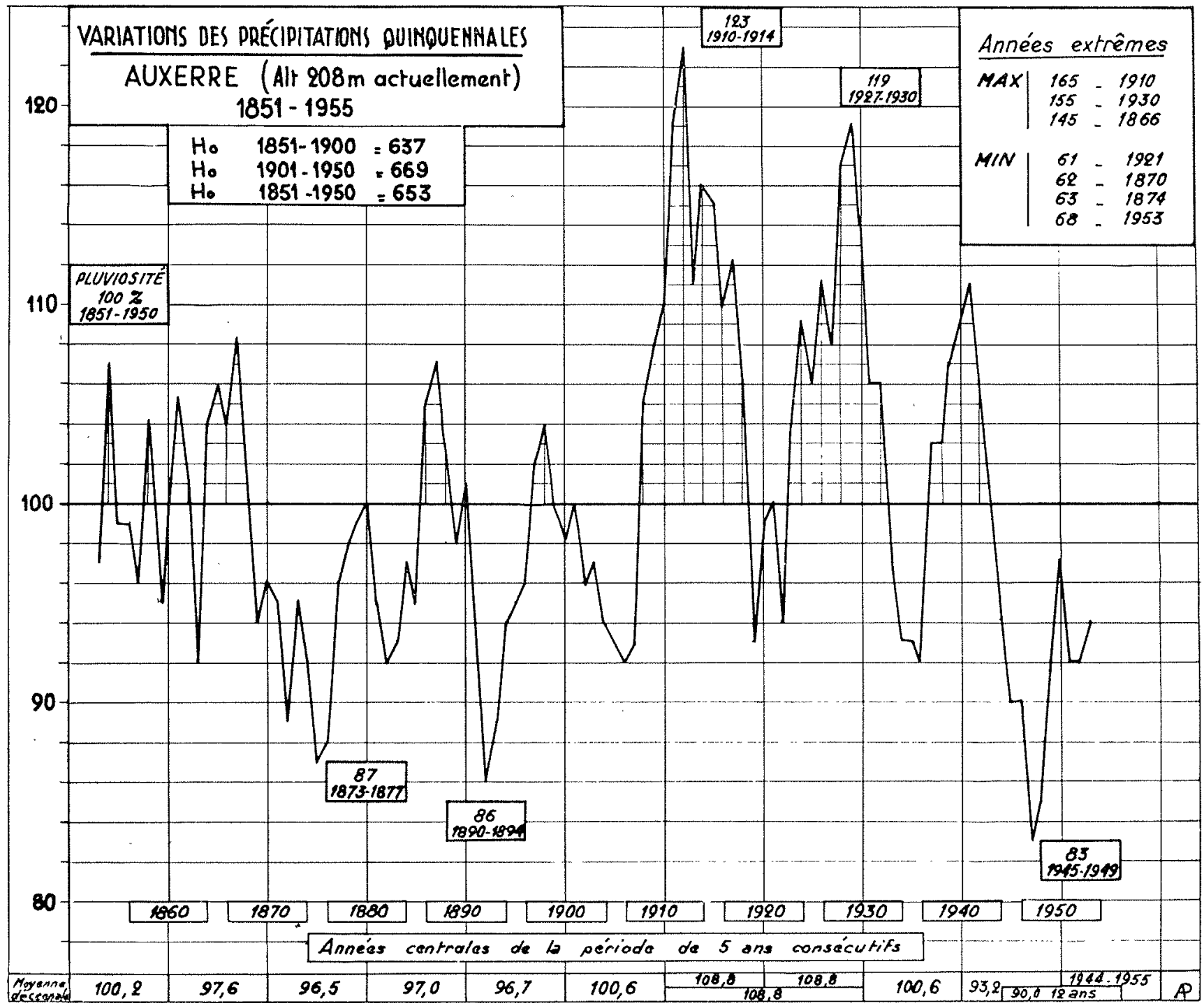

FIG. 1

naire 1851-1950. Le graphique est établi d'après des moyennes quinquennales mobiles. On a encadré quelques séries quinquennales remarquables. En bas du graphique, on a porté les moyennes décennales fixes.

\section{A noter :}

- l'abondance remarquable des 20 ans 19101930, succédant à 40 ans de déficit 1860-1900 et précédant le déficit des années 40 ;

- l'absence de 1949 dans les années minima.

La corrélation entre les débits de la Seine et les précipitations d'Auxerre pour les 30 ans 1927-1956 est de la formule :

$$
\begin{aligned}
& \frac{\mathrm{Q}}{\mathrm{Q}_{0}}-1=1,7\left(\frac{\mathrm{H}}{\mathrm{H}_{0}}-1\right) \quad \mathrm{R}=0,88 \\
& \mathrm{Q}=\text { débits } \\
& \mathrm{H}=\text { précipitations }
\end{aligned}
$$

Les indices $(0)$ correspondent à la moyenne de la période, et les lettres sans indice à une année parliculière.

Ce coefficient de corrélation s'améliore si l'on tient compte en plus des précipitations de l'année antérieure:

$\mathrm{R}=0,92$ avec la formule :

$$
\frac{\mathrm{Q}}{\mathrm{Q}_{0}}-1=1,7\left(\frac{\mathrm{H}}{\mathrm{H}_{0}}-1\right)+0,56\left(\frac{\mathrm{H}^{\prime}}{\mathrm{H}_{0}}-1\right)
$$

Le signe (') concerne l'année antérieure. 
Valeurs numériques :

Déficit d'écoulement :

$$
\mathrm{D}=653 \times 1,08-193=512 \mathrm{~mm}
$$

Quotient d'écoulement:

$$
\begin{aligned}
& \mathrm{C}=193 / 705=0,27 \\
& \mathrm{~K}=\mathrm{Q} / \mathrm{H}_{2}=0,39
\end{aligned}
$$

2" Hydraulicités comparées de la SeINe a Paris. - Mesures et Galculs.

La série de 30 ans de mesures à Paris comporte deux périodes à peu près égales, la première particulièrement humide, la seconde particulièrement sèche.
La période humide $1927-1941=15$ ans est présentée dans le graphique $2 . \quad R=0,91$.

Valeurs numériques :

$$
\begin{aligned}
& \mathrm{D}=767-227=540 \mathrm{~mm} \\
& \mathrm{C}=0,29 \\
& \mathrm{~K}=0,40
\end{aligned}
$$

La période sèche $1944-1955=12$ ans.

$$
\mathrm{R}=0,93
$$

Pour la figure 3, les valeurs numériques sont :

$$
\begin{aligned}
& \mathrm{D}=644-158=486 \mathrm{~mm} \\
& \mathrm{C}=0,25 \\
& \mathrm{~K}=0,38
\end{aligned}
$$

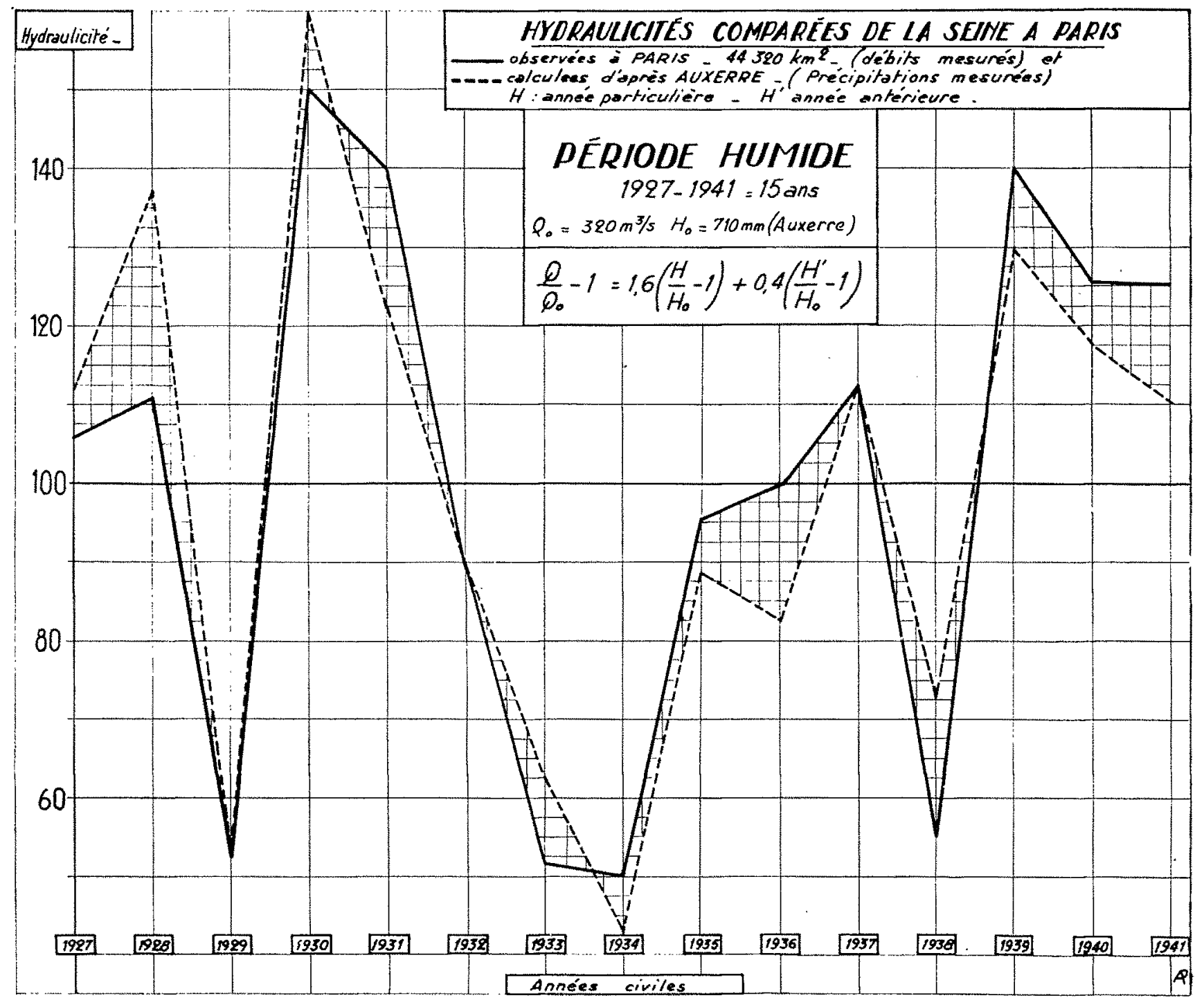

Fic. 2 


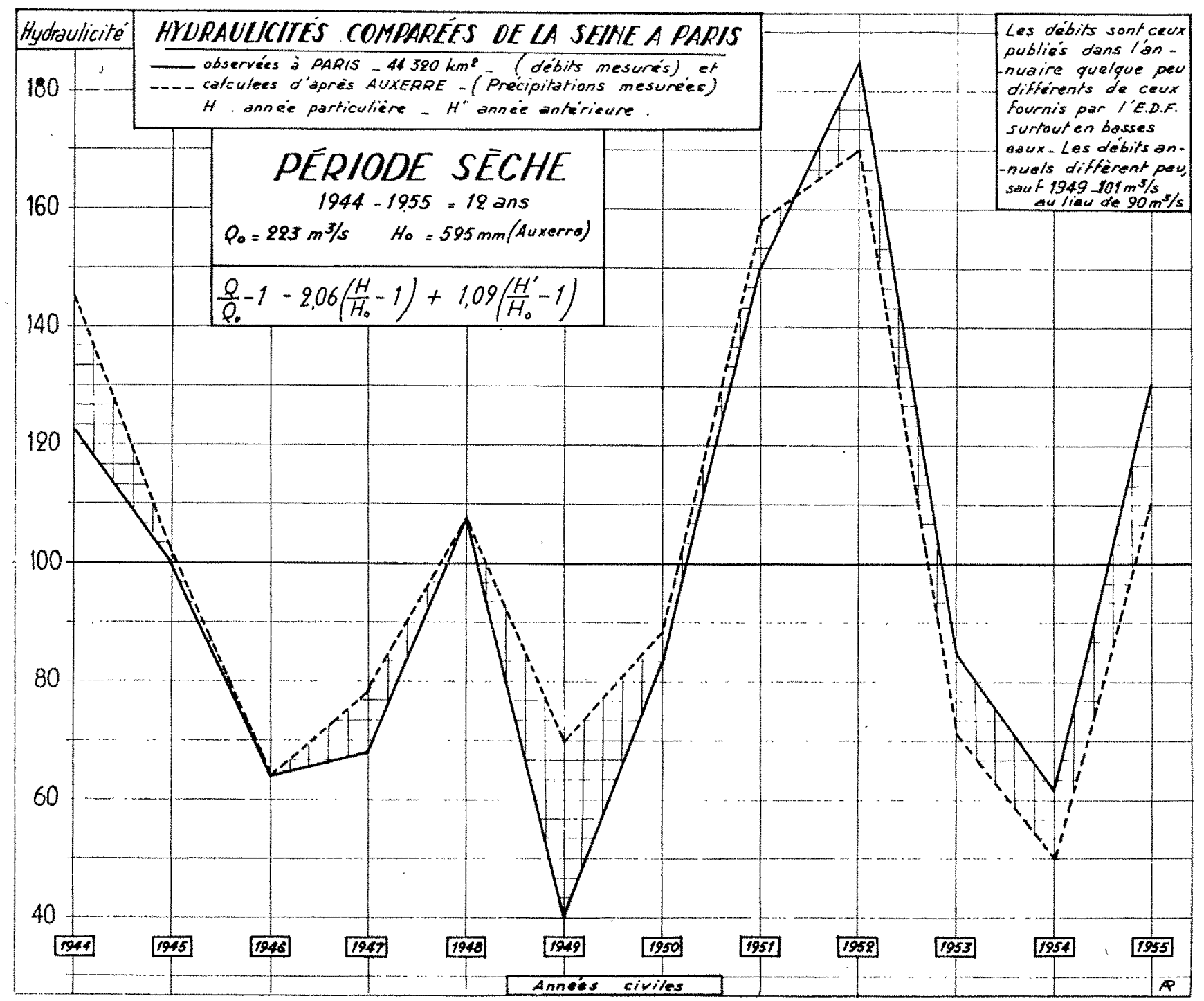

Fig. 3

En période sèche, le déficit est plus faible et l'influence des précipitations de l'année précédente plus marquée qu'en période humide.

$3^{\circ}$ Hydraulicités comparées de la Seine a Melun. - Mesures et calculs poun rat PÉRIODE 1890-1930.

D'après les quatre années communes $1927-$ 1930 , on peut écrire que le module de Paris vaut 1,55 fois celui de Melun, alors que le rapport des bassins versants (BV) est égal à 1,64 .

$4^{\circ}$ Variations du bébit décennal a Paris penDANT UN SIËCLE.

La période unitaire est la décennie (10 années consécutives), chaque point du graphique de la figure 4 représentant une moyenne décennale fixe.

Sur la période centenaire 1851-1950, les débits ont été successivement :

- Calculés d'après Auxerre, avec le coefficient. 1,08 pendant ce siècle, dont on retiendra les 40 années : 1851-1890;

- Rétablis d'après Melun, avec le coefficient 1,55 pendant les 40 années : 1891-1930;

- Mesurés à Paris, directement pendant les 20 dernières années : 1931-1950.

On passe des précipitations aux débits par la formule $\mathrm{Q}=0,66 \mathrm{H}-237$ déduite de la formule indiquée dans le $1^{\circ}$ ) en remplacant $Q_{0}$ et $\mathrm{H}_{0}$ par leur valeur numérique : 193 et $653 \mathrm{~mm}$.

On passe des mm aux $\mathrm{m}^{3} / \mathrm{s}$ en divisant les débits exprimés en mm par 0,71 . 
Débits d'après Auxerre:

$$
Q_{1}=\frac{Q}{0,71}=\frac{Q}{31536 / 44320}=\frac{Q \mathrm{~mm} \times 44320 \mathrm{~km}^{2}}{31536 \mathrm{~s}}
$$

Débits d'après Melun :

$$
\mathrm{Q}_{2}=\mathrm{Q} \text { Melun } \times 1,55
$$

La concordance de $Q_{2}$ avec $Q_{1}$ est particulicrement satisfaisante pour les deux décennies 1891-1900 et 1911-1920.

Débits mesurés directement à Paris $Q_{3}$.

La sćrie centenaire $Q$ est déduite de $Q_{1} Q_{2} Q_{3}$.

Le maximum décennal est 1911-1920.

Le minimum 1941-1950. Le maximum vaut 1,5 fois le minimum.
Quant aux débits annuels extrêmes, nous les estimons par interpolation ainsi :

$$
\begin{aligned}
& 1921 \ldots Q \mathrm{Q}=255 \text { (Loire) } \times 0,31=79 \mathrm{~m}^{3} / \mathrm{s} \\
& 1910 \ldots \mathrm{Q}=2026 \text { (Loire) } \times 0,32=648 \mathrm{~m}^{3} / \mathrm{s}
\end{aligned}
$$

Le coefficient 0,32 résulte des débits observés en 1930 et 1931 à Paris et à Montjean.

D'où un rapport des modules extrêmes égal à 8 environ, comme pour la Loire.

Ces estimations comportent une part d'incertitudes, mais l'intérêt qu'elles présentent suffit à les justifier.

La moyenne trentenaire 1927-1956 et la moyenne centenaire 1851-1950 s'équivalent autour de $275 \mathrm{~m}^{3} / \mathrm{s}$.

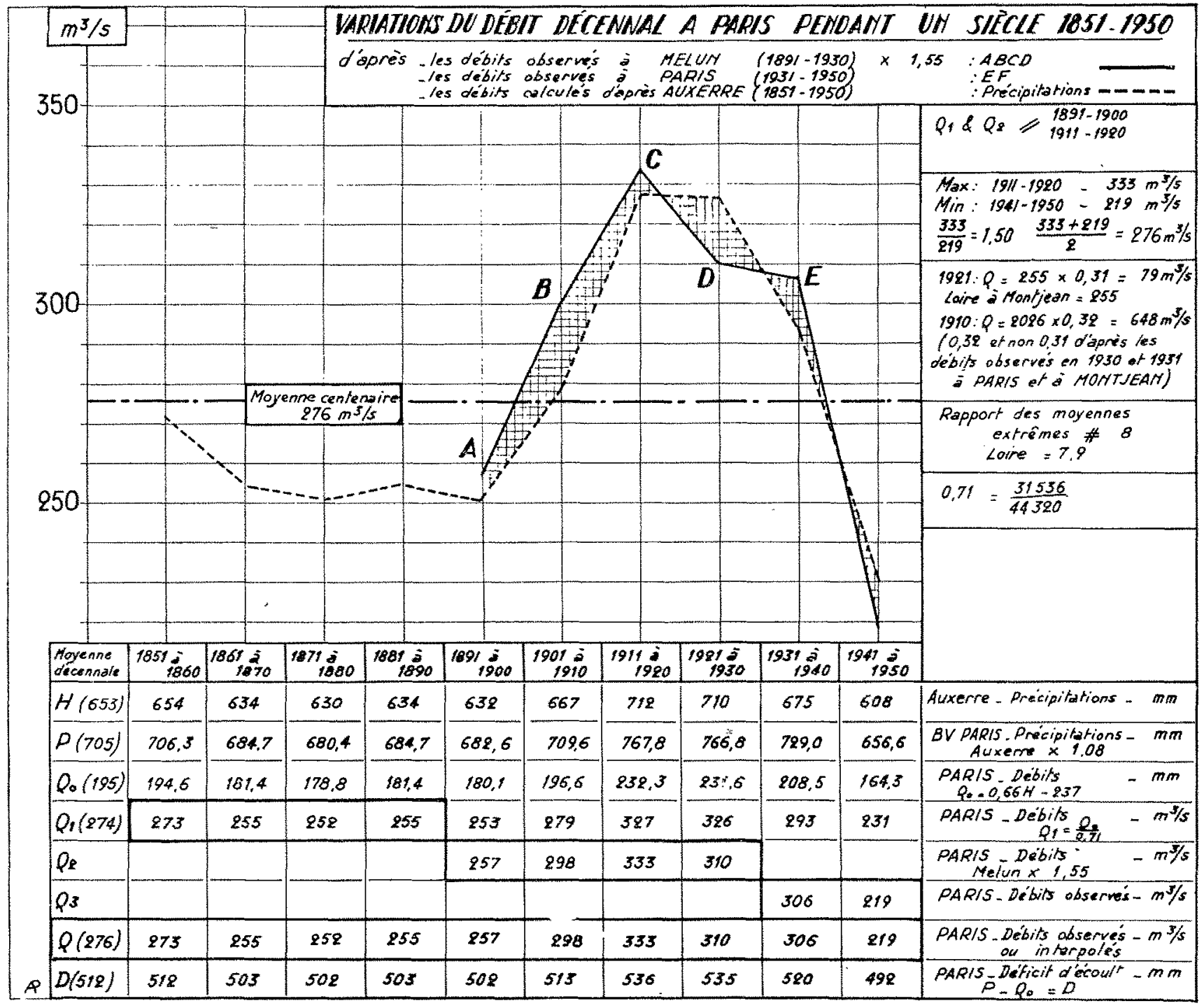




\section{II. - DEBITS DE QUELQUES FLEUVES ET RIVIẼRES FRANÇAIS de 1927 à 1956}

\section{1927-56. DébrTs Moyens Nommaux DE TRÈs LONGUE PÉRIODE.}

Si les précipitations d'Auxerre pendant les trente années 1927-1956 et le siècle 1851-1950 sont équivalentes, d'où par déduction de corrélations les débits de la Seine à Paris pendant ces mêmes périodes, la règle supporte une gé- néralisation. Le tableau II donne pour 15 stations francaises la valeur de ces modules et leur variabilité.

Ces 30 dernières années enregistrent des moyennes annuelles de débits très voisines des moyennes englobant des périodes plus longues, excédant même parfois un siècle (tableau I).

\section{TABLEAU I}

\begin{tabular}{|c|c|c|c|c|}
\hline & $\begin{array}{c}\mathrm{BV} \\
\mathrm{km}^{2}\end{array}$ & $\begin{array}{c}1927-1956 \\
30 \text { ans } \\
\mathrm{m}^{3} / \mathrm{s} \\
\end{array}$ & $\begin{array}{c}\text { Longue } \\
\text { période } \\
\mathrm{m}^{3} / \mathrm{s}\end{array}$ & \\
\hline $\begin{array}{l}\text { Le Rhin à Bâle } \ldots \ldots \ldots \ldots \ldots \ldots \ldots \\
\text { Le Rhin à Rheinfelden } \ldots \ldots \ldots \ldots \ldots\end{array}$ & $\begin{array}{l}35925 \\
34550\end{array}$ & 1026 & 1026 & $1808-1957=150 \mathrm{ans}$ \\
\hline La Loire à Montjean........... & 110000 & $879 ?$ & $843 ?$ & $1880-1949=70$ ans \\
\hline L'Isère à Beaumont. . . . . . . . . . . . . & 11500 & 324 & $\begin{array}{l}317 \\
330\end{array}$ & $\begin{aligned} 1920-1956 & =37 \text { ans } \\
1904-1956 & =53 \text { ans }\end{aligned}$ \\
\hline La Durance à Mirabeau.............. & 11920 & 186 & 191 & $1904-1956=53$ ans \\
\hline
\end{tabular}

2。 1927-56. PÉRIOde TRÉS CONTRASTÉE.

Loire : écart moyen : $35,6 \%$ contre $30,9 \%$ (1880-1949).

Les écarts d'hydraulicité sont d'autant plus grands que le bassin est moins arrosé. Ainsi le Rhône à Génissiat n'a que 11,9 \% d'écart moyen contre $35,6 \%$ pour la Loire. Avec un écart moyen de $33,7 \%$, la Seine a une « variabilité annuelle $»$ du même ordre de grandeur que la Loire : ce n'est pas un cours d'eau pondéré, ce qu'explique sa faible pluviosité, les fluctuations pluviales étant d'autant plus grandes que les précipitations sont faibles.

3० 1927-1956. SÉRIE QUI COMPORTE DEUX PÉRIODES SUCCESSIVES DF 15 ANS NETTEMFNT DIFFERENCIÉES, LA PREMIÈRF EXCÉDENTAIRE, LA SECONDE DÉFICITAIRE.

C'est ce qui ressort nettement du tableau II. L'écart entre les deux hydraulicités de ces deux périodes est d'autant plus grand que le bassin est moins arrosé et moins nival (ou glaciaire).

Ainsi le Rhône à Génissiat (glaciation $=9 \%$ ) et le Rhin à Bâle (glaciation $=1,5 \%$ ) ont entre ces deux périodes un rapport d'hydraulicilé de 1,09 et 1,10 ; ce mème rapport monte à 1,44 pour la Seine et 1,67 sur la Loire.

\section{1927-1956. ANNÉEs EXTrÊMES : 1930 MAXIMUM, 1949 MINIMUM.}

Toutefois les débits de 1930 ont été inférieurs à ceux de 1910.

Et ceux de 1949 supérieurs à ceux de 1921 , sauf dans le Sud-Ouest, où 1925 a été plus déficitaire en 1921.

Ainsi sur la Loire :

$$
\begin{array}{r}
1910 \ldots .2026 \mathrm{~m}^{3} / \mathrm{s} \text { contre } 1563 \text { en } 1930 \\
1921 \ldots .253 \mathrm{~m}^{3} / \mathrm{s} \text { contre } 293 \text { en } 1949
\end{array}
$$

La période sèche de 15 ans a comporté des années particulièrement chaudes qui ont entraîné la dégradation des réserves glaciaires dans les Alpes, en compensant la pénurie pluviale, el auğmenté le déficit d'écoulement dans les bassins à régime pluvial. En particulier, l'année 1949 a dépassé de $1,5^{\circ}$ la moyenne centenaire.

Il faudrait surtout vérifier que la saison chaude, époque de Ia fusion, juin-juillet-août, a 
TABLEAU II

1927-1956 : DÉBITS ANNUELS, EN FRANCE, ET LEUR VARIABILITÉ

\begin{tabular}{|c|c|c|c|c|c|c|c|c|c|c|c|c|c|c|c|}
\hline \multirow[b]{2}{*}{ Bassins } & \multicolumn{2}{|c|}{$-1-$} & \multicolumn{3}{|c|}{$-2=$} & \multirow{2}{*}[\begin{array}{c}{-3.}\\
{\%}\end{array}]{} & \multicolumn{2}{|c|}{$-4-$} & \multicolumn{2}{|c|}{$-5-$} & \multicolumn{2}{|c|}{$-6-$} & \multicolumn{2}{|c|}{$-7-$} & \multirow{2}{*}{$\begin{array}{c}\text { Bassins } \\
\text { principaux }\end{array}$} \\
\hline & $\mathrm{km}^{2}$ & $\begin{array}{c}\text { Altitude } \\
\text { moyenne } \\
\text { m }\end{array}$ & $\begin{array}{c}\mathrm{Q} \\
\mathrm{m}^{3} / \mathrm{s}\end{array}$ & $\begin{array}{c}q \\
1 / s / \mathrm{k}^{2}\end{array}$ & $\begin{array}{c}Q^{\prime} \\
\mathrm{mr}\end{array}$ & & Max. & Année & Min. & Année & $\begin{array}{c}1927- \\
1941 \\
h_{1}\end{array}$ & $\mid \begin{array}{c}1942- \\
1956 \\
h_{2}\end{array}$ & $h_{1} / h_{2}$ & $\mathrm{H}_{\text {moy }}$ & \\
\hline Rhin Suisse............ & 34550 & 1100 & 1026 & 29,7 & 937 & 13,7 & 127 & 1939 & 62 & 1949 & 105 & 95 & 1,10 & 1425 & Rhin \\
\hline Rhône à Génissiat........ & 10900 & - & 361 & 33,1 & 1045 & 11,9 & 132 & 1930 & 71 & 1949 & 104,5 & 95,5 & 1,09 & 1500 & \\
\hline Rhône au Teil.......... & 70000 & - & 1484 & 21,2 & 669 & 21,8 & 144 & 1930 & 55 & 1949 & 112,6 & 87,4 & 1,29 & 1200 & \\
\hline Isère à Beaumont $\ldots \ldots \ldots$. & 11500 & 1330 & 324 & 28,0 & 885 & 18,6 & 143 & 1951 & 55 & 1949 & 106,3 & 93,7 & 1,13 & 1400 & Rhône \\
\hline Jurance à Mirabeau........ & 11900 & 1290 & 186 & 15,7 & 490 & 23,7 & 190 & 1936 & 60 & 1944 & 110 & 90 & 1,22 & 1100 & \\
\hline Ain $\grave{a}$ Cize-Bolozon....... & 2560 & 720 & 96 & 37,5 & 1182 & 23,9 & 170 & 1935 & 59 & 1953 & 106 & 94 & 1,13 & 1750 & \\
\hline Fier à Vallières.......... & 1350 & 1350 & 41,3 & 30,6 & 965 & 26,9 & 167 & 1930 & 46 & 1949 & 114 & 86 & 1,33 & 1500 & \\
\hline 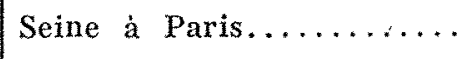 & 44300 & - & 271,7 & 6,12 & 194 & 33,7 & 170 & 1930 & $3 \pi$ & 1949 & 118 & 82 & 1,44 & 700 & Seine \\
\hline Loire à Montjean . . . . . . . . & 110000 & - & $879(?)$ & 8,0 & 252 & 35,6 & 170 & 1930 & 33 & 1949 & 125 & 75 & 1,67 & 770 & \\
\hline Loire à Gien............ & 36000 & - & 360 & 10,0 & 315 & 34,5 & 161 & 1930 & 33 & 1949 & 123 & 77 & 1,60 & 850 & Loire \\
\hline Creuse à Eguzon.......... & 2400 & 445 & 29,7 & 12,4 & 390 & 34,3 & 165 & 1930 & 29 & 1949 & 123 & 77 & 1,60 & $92 \tilde{3}$ & \\
\hline Dordogne à Cénac........ & 8700 & 610 & 178 & 20,5 & 645 & 27,8 & 151 & 1930 & 45 & 1953 & 116 & 84 & 1,38 & 1150 & Dordogne \\
\hline Dordogne $\dot{a}$ Argentat..... & 4. 400 & 775 & 100 & 22,7 & 715 & 28,1 & 159 & 1930 & 43 & 1949 & 117 & 83 & 1,41 & 1200 & \\
\hline Garonne à Mas-d'Agenais... & 52000 & - & $546(?)$ & $10,5(?)$ & $331(?)$ & 28,0 & 163 & 1930 & 35 & 1949 & 117 & 83 & 1,41 & 900 & Garonne \\
\hline l ot à Cajare............. & 7000 & 845 & 125,3 & 17,9 & 564 & 27,8 & 166 & 1935 & 29 & 1949 & 119 & 81 & 1,47 & 1100 & \\
\hline $\begin{array}{l}\text { (1) Superficie et altitu } \\
\text { (2) Débits en } \mathrm{m}^{3} / \mathrm{s}, 1 / \mathrm{s} \\
\text { (3) Ecarts mogens en }\end{array}$ & $\begin{array}{l}\text { moyenn } \\
\mathrm{km}^{2} \text { et en } \\
\text { du débi }\end{array}$ & min. & $(7$ & Précipito & Ions (ordr & e de & ndeur & $\begin{array}{l}\text { (4) } \mathrm{HIJ}_{\mathrm{I}} \\
\text { (5) } \mathrm{Hy} \\
\text { (6) } \mathrm{HI}_{\mathrm{y}} \\
\text {. }\end{array}$ & $\begin{array}{l}\text { drantic } \\
\text { draulic } \\
\text { draulic }\end{array}$ & $\begin{array}{l}\text { ité } \max \\
\text { ité } \min \\
\text { ités } 192\end{array}$ & $\begin{array}{l}\text { ximum, } \\
\text { iimum, } \\
27-1941\end{array}$ & $\begin{array}{l}\text { et ann } \\
\text { et ann } \\
\text { et } 194\end{array}$ & $\begin{array}{l}\text { ée cor } \\
\text { lée cor } \\
2-1956\end{array}$ & $\begin{array}{l}\text { espond } \\
\text { espond } \\
\text { et rapp }\end{array}$ & $\begin{array}{l}\text { lante. } \\
\text { anie. } \\
\text { port. }\end{array}$ \\
\hline
\end{tabular}




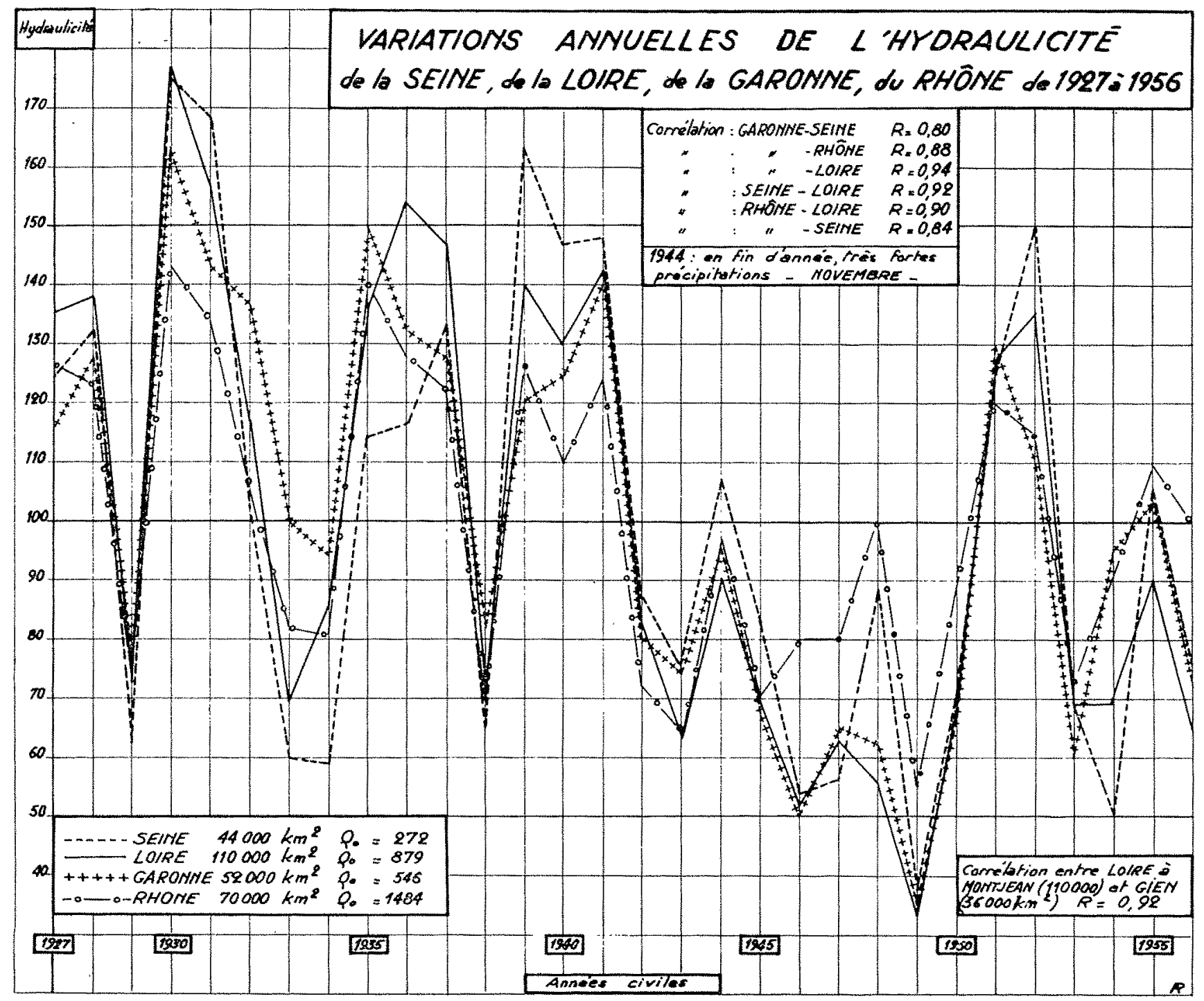

FIE. 5

été plus chaude que la moyenne des températures de ces mêmes mois: ce ne fut pas toujours le cas ces dernières années.

$5^{\circ}$ Les précipitations portées à l'avant-dernière colonne du tableau II ne sont qu'indicatives. Elles ont été le plus souvent estimées d'après un déficil d'écoulement admis a priori.

L'absence de statistiques pluviales, par bassins versants, ne permet pas de fixer avec plus d'exaclilude le déficit d'écoulement et ses variations locales d'après les différentes régions francaises. Grosso modo, nous estimons le déficit moyen à $510 \mathrm{~mm}$ pour le bassin de la Seine, à 525 pour celui de la Loire, à 540 pour la Garonne, et à 530 (peut-être même 550) pour le Rhône au Teil. En tout cas, des 3 facteurs : Précipitations $(\mathbf{H})$, Ecoulements (Q) et Evaporations (E), ce dernier cst de beaucoup le moins variable. $6^{\circ}$ 1927-1956. VARIATIONS ANNUELLES DE L'HYDRAULICITÉ DANS LES GRANDS BASSINS FraNÇAIS (fig. 5).

Entre la Loire - qui occupe dans notre pays une situation centrale - et les 3 autres grands fleuves français, la Seine, la Garonne et le Rhône, les coefficients de corrélation sont assez élevés :

$$
\begin{array}{ll}
\text { Loire-Seine . . . . . } & R=0,92 \\
\text { Loire-Garonne . . . } & R=0,94 \\
\text { Loire-Rhône . . . . } & R=0,90
\end{array}
$$

Nous terminons par les graphiques de distribution de fréquence, avec ajustement de Ia loi de Galton, et des diagrammes des fréquences partielles sur les 70 ans de 1887 à 1956 , des précipitations d'Auxerre et des modules de la Seine et de la Loire (fig. $6,7,8$ ). 
DISTRIBUTION DE FREQUENCE DES PRECIPITATIONS ANNUELLES D'AUXERRE

$1887-1956=70$ ans

Courbe de répartition empirique. Loi de Galton.

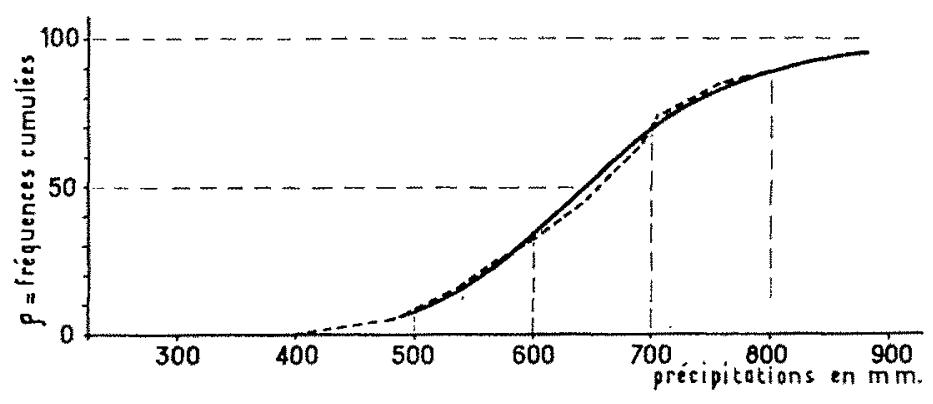

DIAGRAMME DES FREQUENCES PARTIELLES

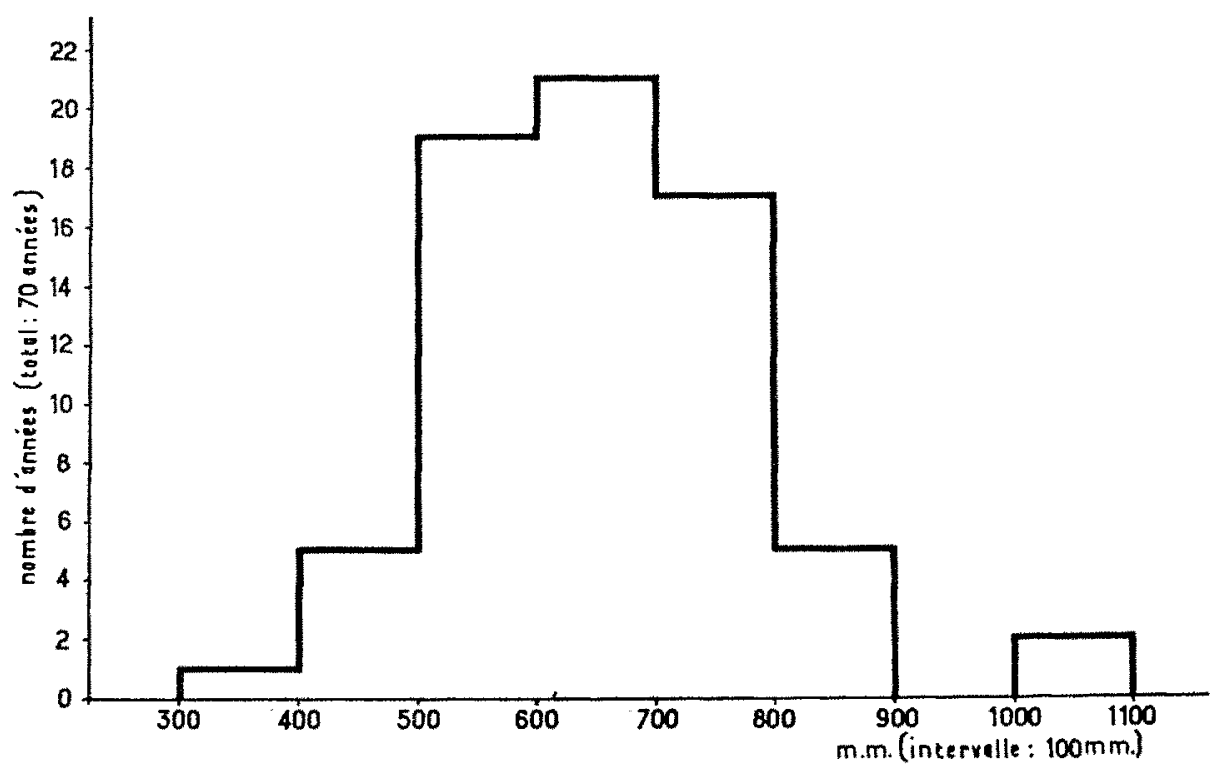

Fig. 6
DISTRIBUTION DE FREQUENCE DU DEBIT ANNUEL DE LA SEINE A PARIS

$1887-1956=70$ ans

-.... Courbe de répartition empirique.

Loi de Galton.

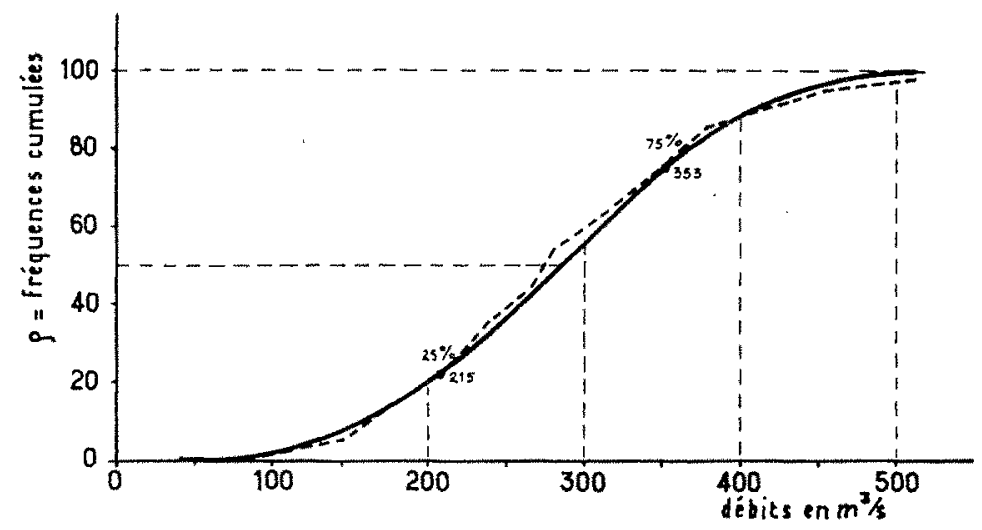

DIAGRAMME DES FREQUENCES PARTIELLES

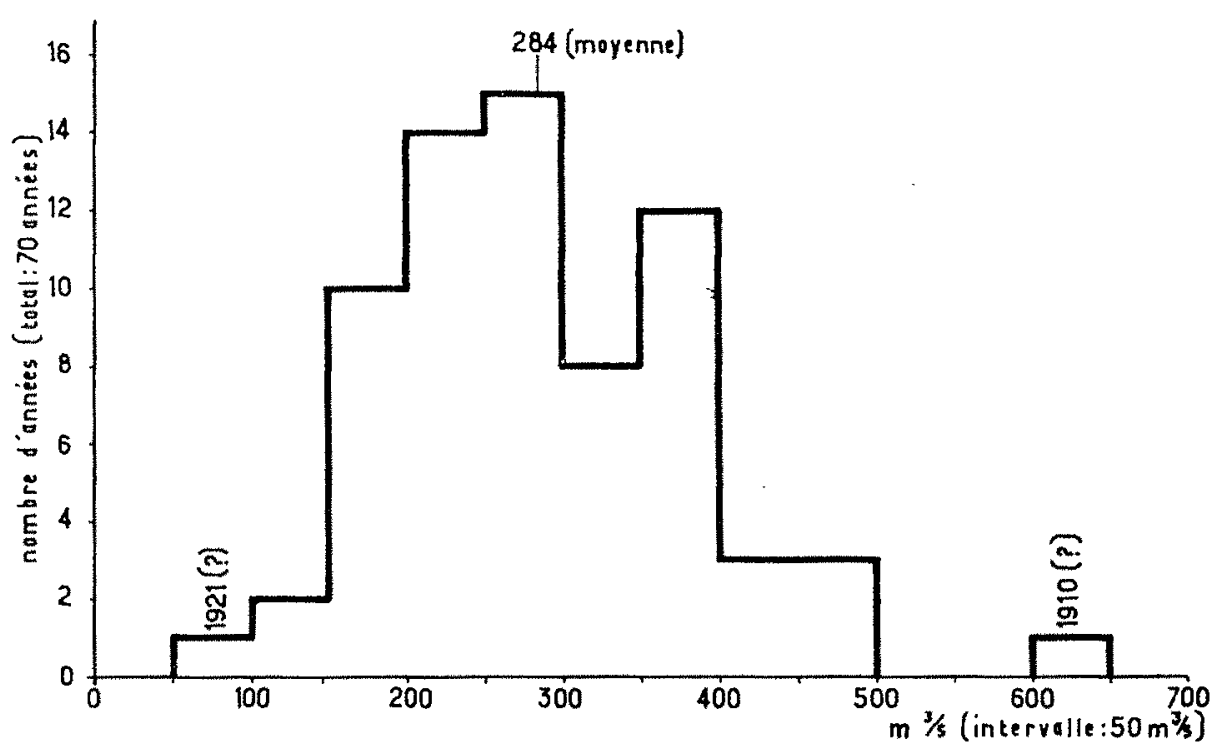

Fig. 7 

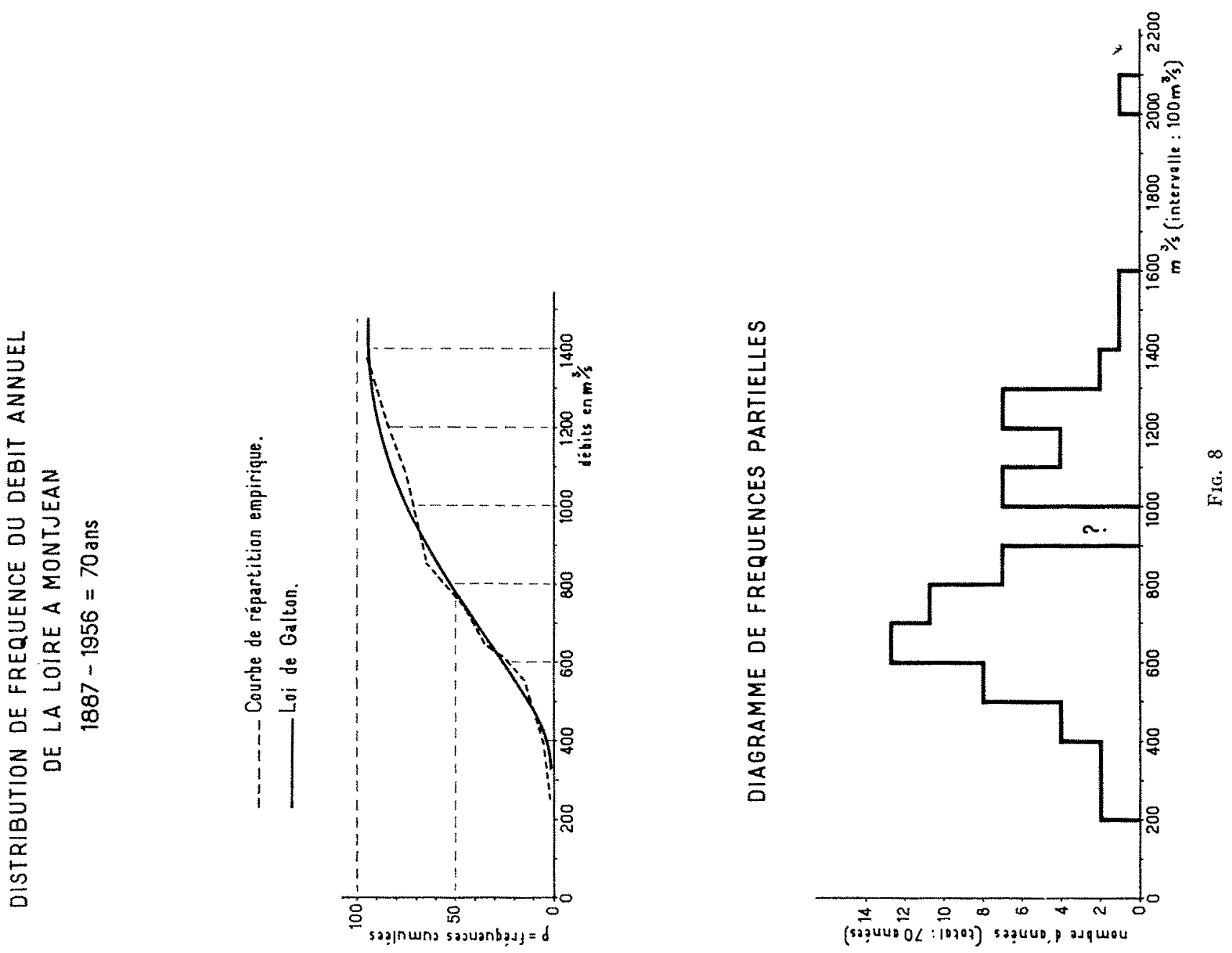

Cet article condense quatre études que l'on pourra trouver à la Société Hydrotechnique de France, 199, rue de Grenelle, Paris (VII').

\section{COMMENTAIRE}

président: M. Dermiñ

M. le Président remercie M. Coutagne pour sa vaste synthèse sur les variations de l'hydraulicité en France et $M$. DE BEaunegard d'avoir su dégager de cette synthèse les éléments essentiels et de les avoir rendus assimilables, dans le temps qui lui était imparti. 